\title{
A group from Hackenbush game
}

\author{
Awad Alabdala* (1) and Essam El-Seidy
}

\author{
* Correspondence: \\ awadtalalalabdal@hotmail.com \\ Department of Mathematics, Faculty \\ of Science, Ain Shams University, \\ Cairo, Egypt
}

\begin{abstract}
This research presents the reader some algebraic operations related to combinatorial games and gives a detailed outlook of a special game called Hackenbush game. We will deduce a group with a special feature with the help of some basic algebraic concepts. A fresh outlook to some combinatorial mathematical algebraic operations and concepts through the evaluation of a deduced group from this game.

Keywords: Combinatorial games, Hackenbush game, Groups, Graph theory, Hackenbush group, Moving to graph

AMS Subject Classification: 91Axx, 20Nxx, 05Cxx
\end{abstract}

\section{Introduction}

As a result of the many mathematical research done by Lagrange, Abel, Galois, and others in the fields of geometry, number's theory, and algebraic equations at the end of the eighteenth century, early nineteenth century, the mathematical theory of "groups" was discovered [1-3]. That theory has led researchers to the discovery of many essential mathematical concepts such as "sub-groups," "graphs and groups," and plenty others. By building on previous credited works and researches, we can express mathematical groups as graphs. By analyzing the structures of graphs, we can deduce the properties of their related groups, which can be done by exploiting the notion of "identity" in "group theory."

By expressing groups by their associated graphs, or in other words "identity graphs," we can utilize the "identity graphs" theory's equations and concepts to conclude the following.

Two elements $\mathrm{x}, \mathrm{y}$ in a group are adjacent or can be joined by an edge if $x . y=e(e$, identity element of $G$ ).

Since we know that $x, y=y . x=e$, there is no need to utilize the property of commutatively. It is by convention; every element is adjoined with the identity of the group $G$. This is the convention we use when trying to represent a group by a graph. The vertices correspond to the elements of the group, hence the order of the group $G$ corresponds to the number of vertices in the identity graph $[4,5]$.

For example, see the identity graph for some groups in Figs. 1 and 2.

In this research, we will utilize the concepts of "graph theory" to analyze some combinatorial mathematical algebraic operations and concepts through the evaluation of a deduced group from a combinatorial game called "Hackenbush game." 


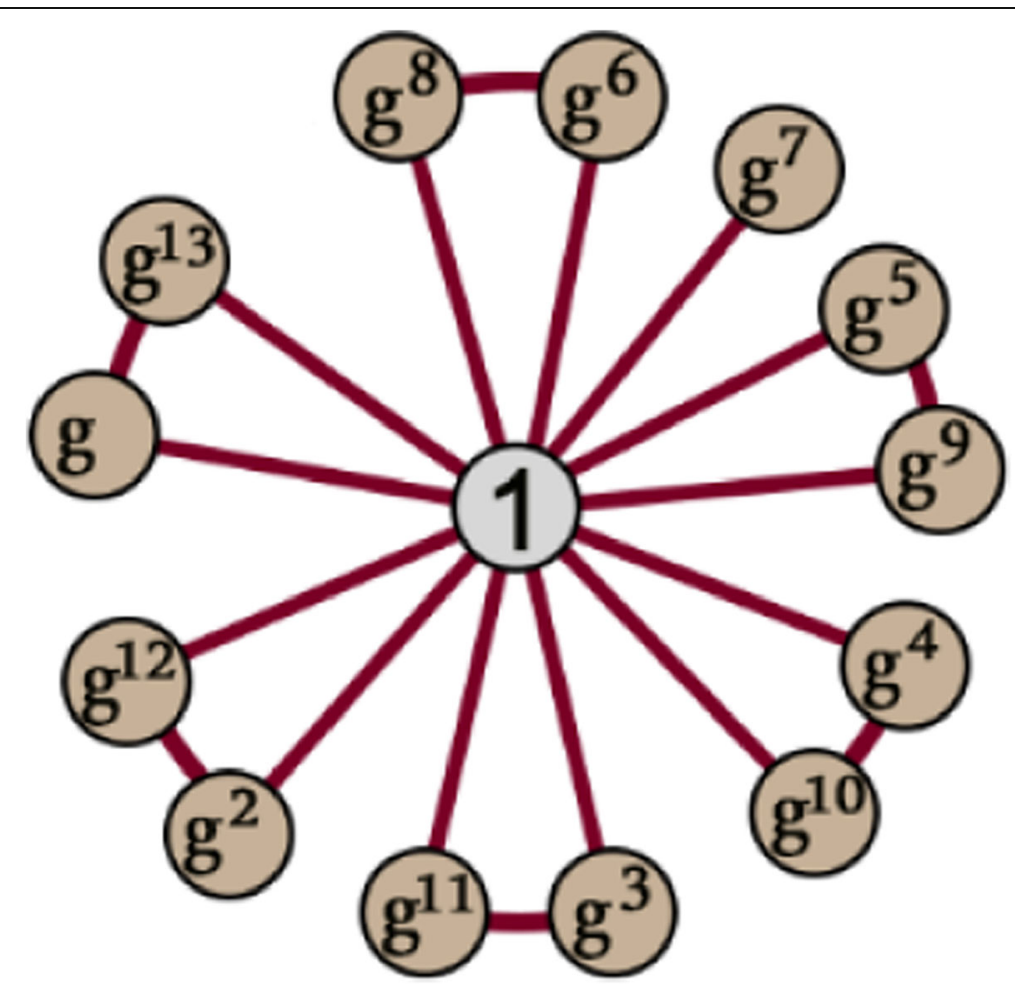

Fig. 1 An identity graph for the cyclic group $G=\left\langle g \mid g^{14}=1\right\rangle$

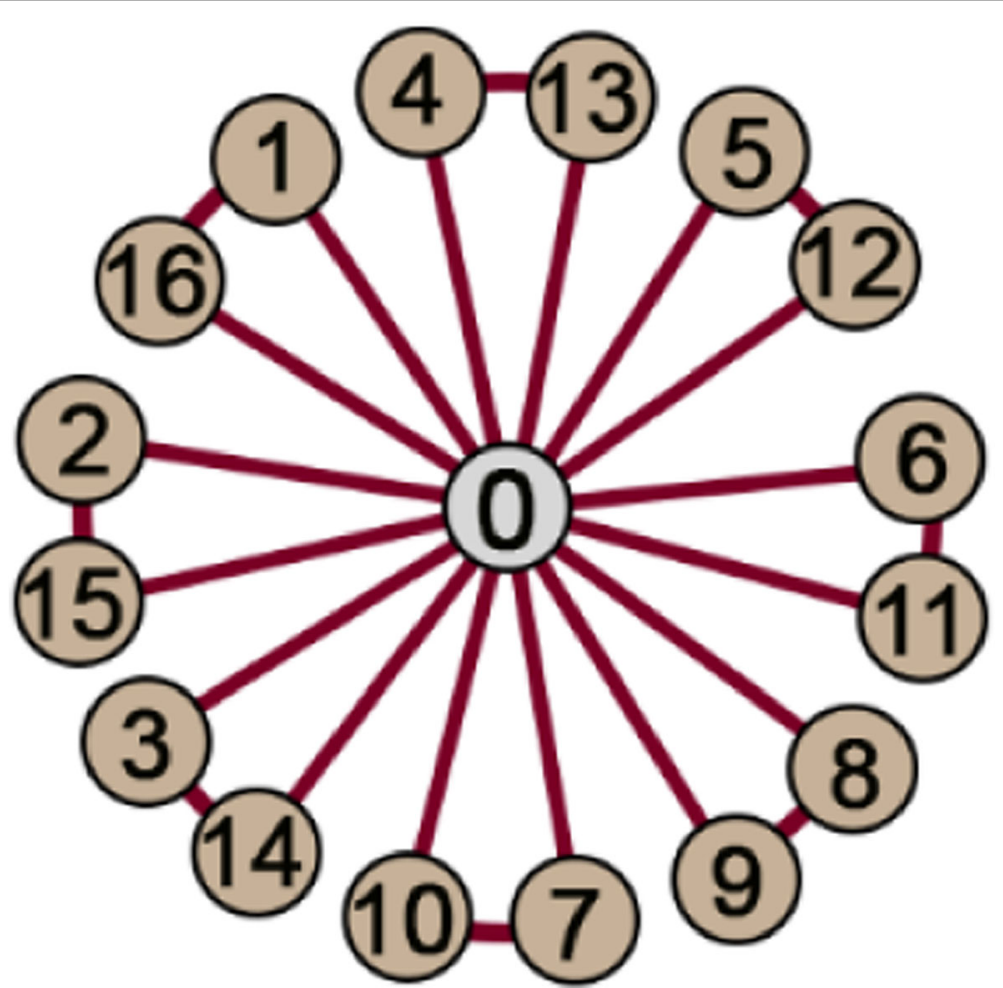

Fig. 2 An identity graph for the $Z_{17}=\{0,1,2, \ldots, 16\}$ the group under addition modulo 17 


\section{Combinatorial games theory}

"Combinatorial game theory" describes the study of sequential games with perfect information. When playing "Hackenbush," all players know all the possible outcomes from a given position with no randomness [6]. The game is defined with the following attributes:

(A) There are two players, left and right.

(B) There is always a finite number of positions, in addition to a starting position.

The game is played by the following rules:

(C) Left and right alternate making moves.

(D) Both players have access to all information always.

(E) There is no randomness to moves made, such as rolling a dice.

(F) A player loses when he/she can no longer make any legal moves.

(G) The game ends when the ending condition is met.

If we have a game $G$ and this game includes options for player $\mathrm{L}$ named $G_{L}$ and options for player R named $G_{R}$.

Now, we will try to explain the manner of dealing with the games, as well as showing its rules.

$$
\text { (A) } G=\left\{G_{L} \mid G_{R}\right\}
$$

Numbers represent the number of free moves or the moves possible to a particular player. By convention, positive numbers represent an advantage for left, while negative numbers represent an advantage for right. They are defined recursively with 0 being the base case.

(B) $0=\{\mid\}$ we will explain Zero game in detail in the section "Hackenbush game".

(C) $G=0$ means the first player loses

(D) $1=\{0 \mid\}, 2=\{1 \mid\}, 3=\{2 \mid\}$

(E) $-1=\{\mid 0\},-2=\{\mid-1\},-3=\{\mid-2\}$

Star, written as * or $\{0 \mid 0\}$, is the first player's wins since either player must move to a Zero game, which will be defined later in the research, therefore wins.

$*+* 0$, because the first player must turn one copy of " to a 0 , and the other player will have to turn the other copy of " to a 0 as well, at this point, the first player would lose, since $0+0$ admits no moves.

Like all other games, the game * is neither positive nor negative, in which the first player wins.

$(\mathrm{F}) *=\{0 \mid 0\}$

(G) * $=-*$

(H) $G \| 0$ means the second player loses

(I) $G>0$ means left wins 


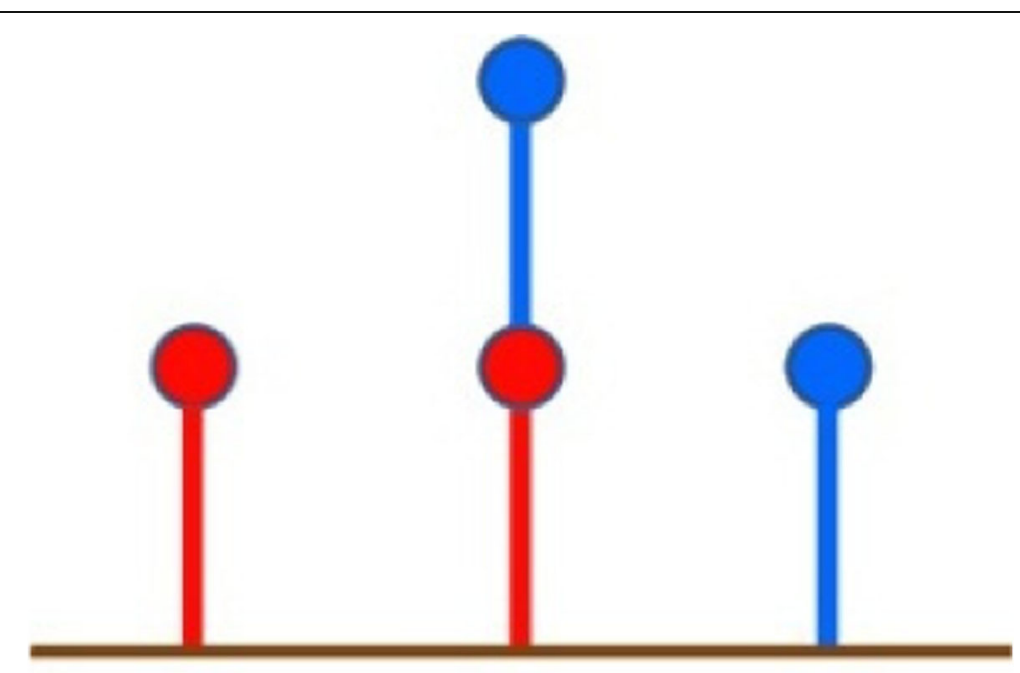

Fig. 3 An example of Hackenbush game

(J) $G<0$ means right wins

Up, written as $\uparrow$, is a position in combinatorial game theory [7]. In standard notation,

$(\mathrm{K}) \uparrow=\{0 \mid *\}$

Down, written as $\downarrow$, is a position in combinatorial game theory [7]. In standard notation,

(L) $\downarrow=\{* \mid 0\}$

(M) $\downarrow=-\uparrow$

We will explain the last two definitions in detail in the next section.

\section{Hackenbush game}

In Hackenbush, the drawn figure uses vertices and line segments, which are connected to a final line called the ground. Players take turns deleting one of their lines.

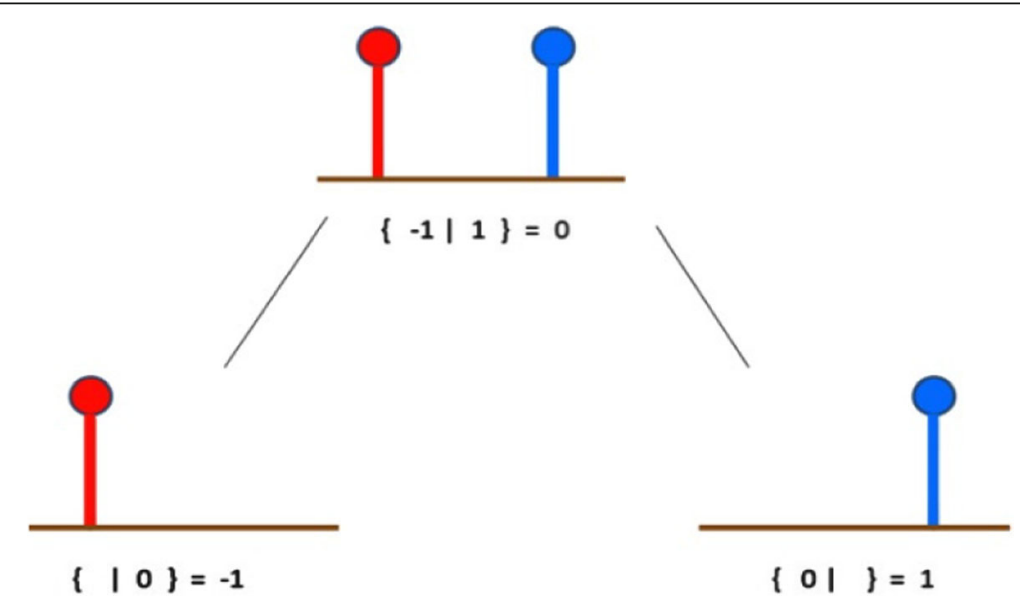

Fig. 4 A simple Hackenbush game tree with values 


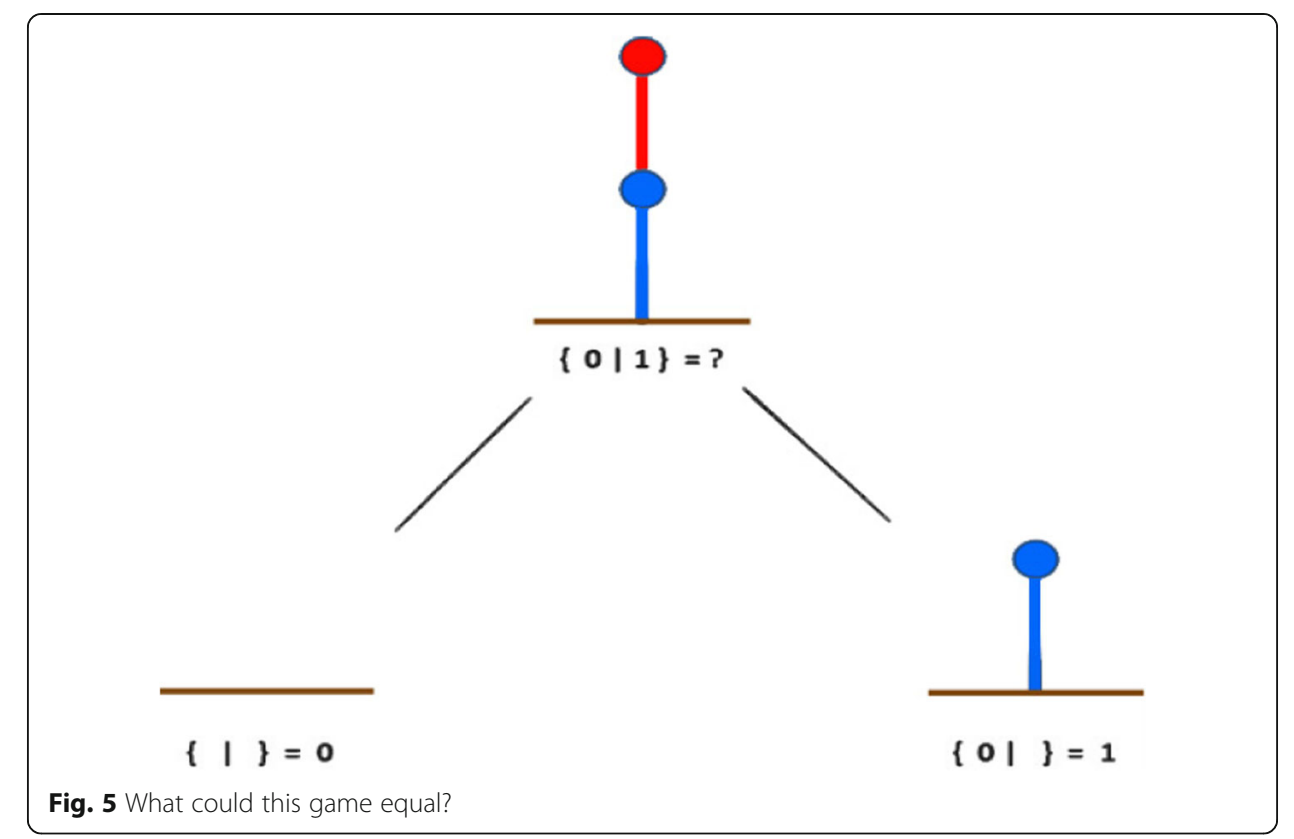

Therefore, left and right take on the colors blue and red respectively. If there is, at any time, a path that cannot be drawn from the ground to a line segment, the segment is deleted. This allows for more strategic plays as a player can delete an opponent's move during their turn $[7,8]$. Figure 3 is an example of the Hackenbush game.

Now, we will discuss how the game is played. In a game represented by Fig. 3. We will pick left to make the first move. Left has two legal moves. He can either remove the line on the right or the line on the top of the red line. However, the latter move is best, because it allows right to take his middle piece, and effectively remove left's piece with it. Likewise, right should take his middle piece if he moves first for that same exact reason. That would be the optimal plays in that current example. Mathematically,

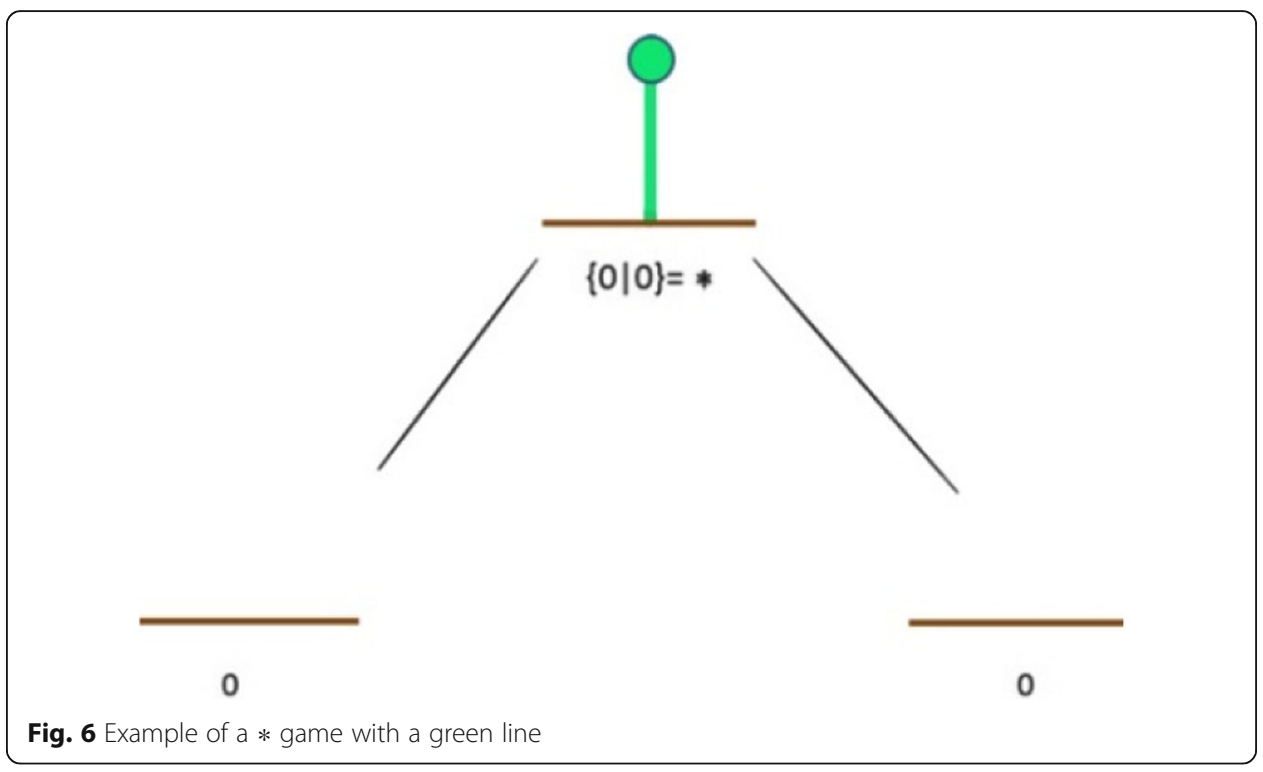




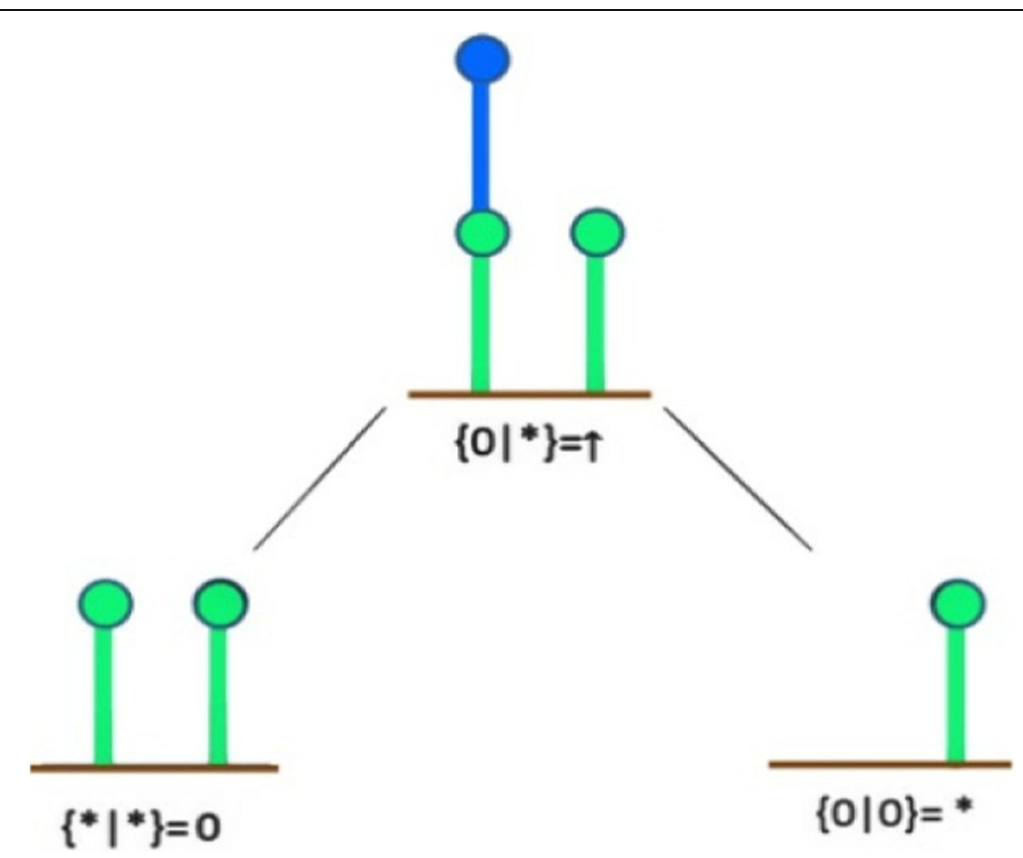

Fig. 7 An example of an up game

after every move, the resulting game's board becomes a sub-game of the original game's board, or in other words, a new game with new independent possibilities $[9,10]$.

Assuming optimal plays are always being made, finite values can be assigned to each game or sub-game. These values are determined by observing the advantages the left player has, after each move. For example, after Left makes a move, he has a moves advantage over right, but after right moves, left has $b$ moves advantage over right.

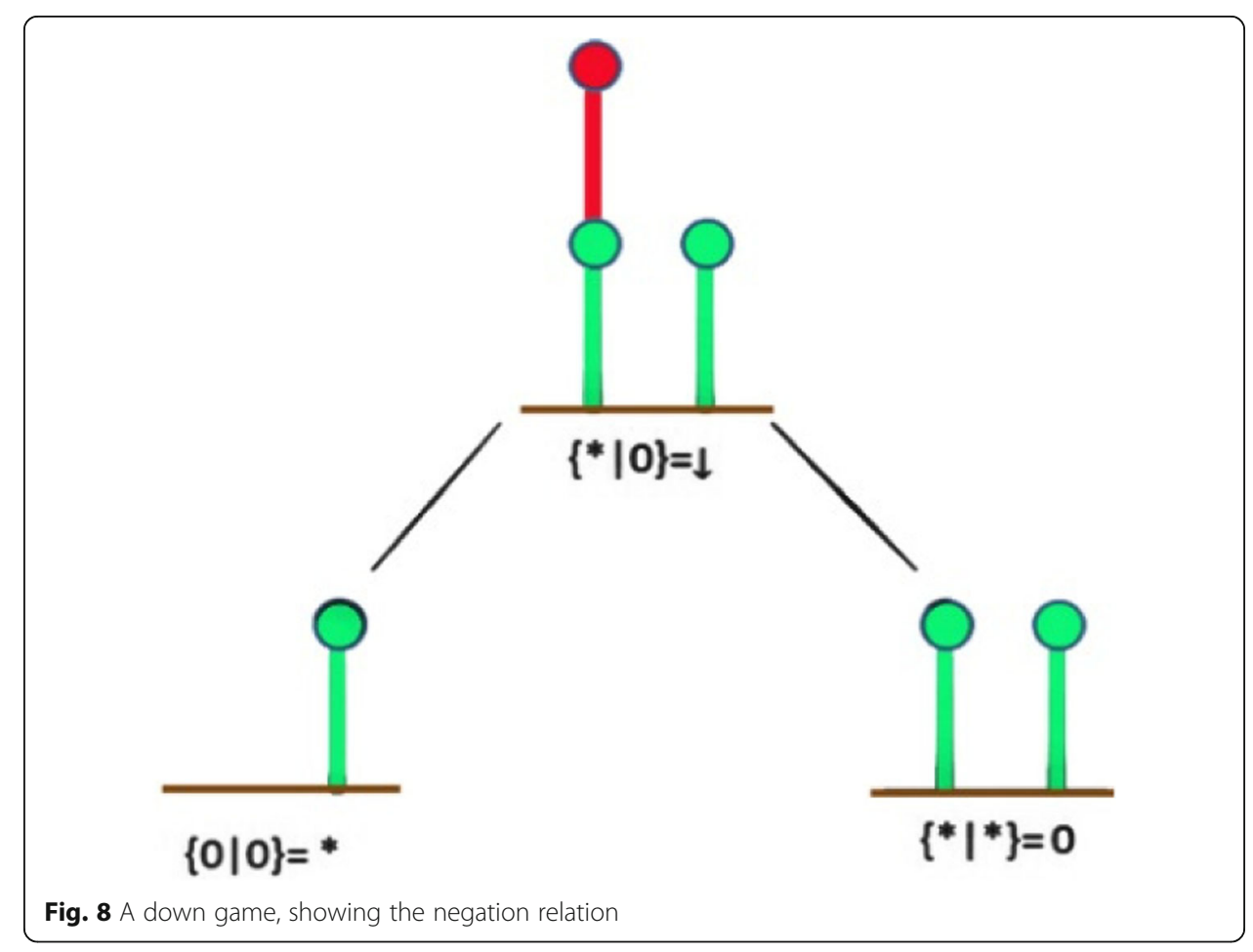




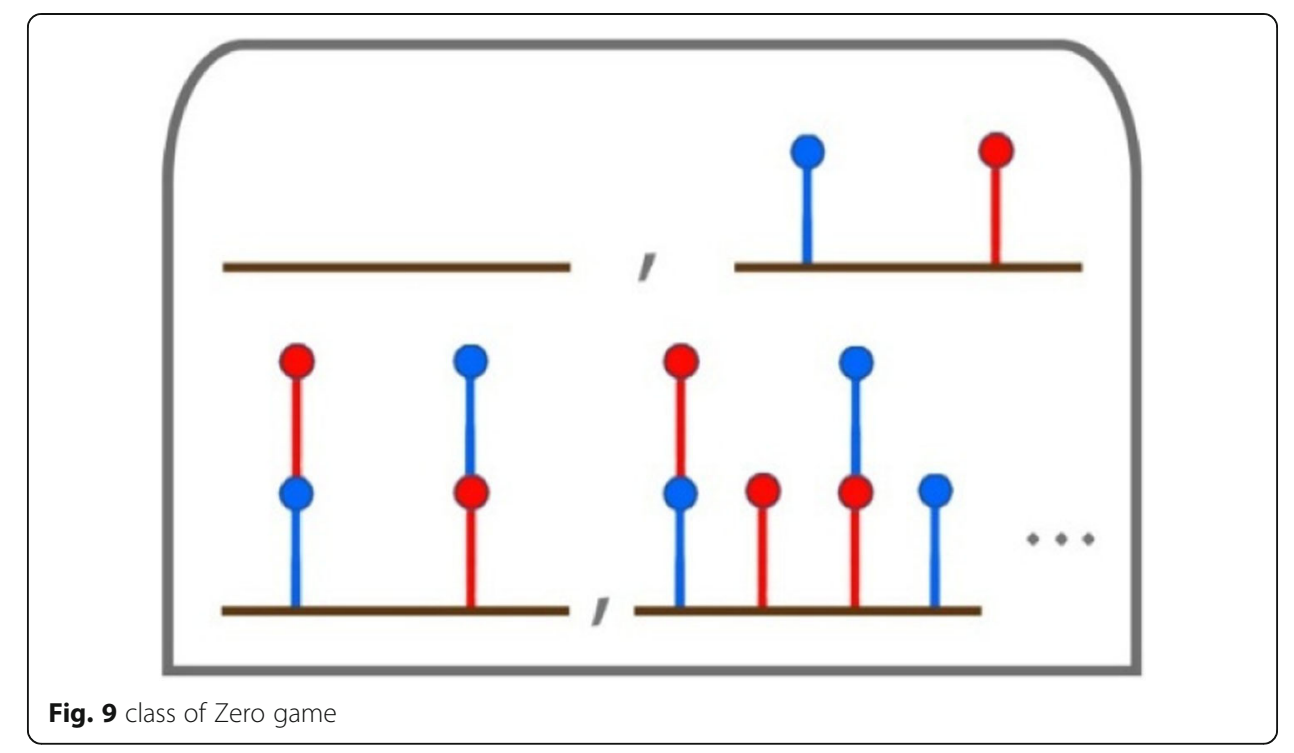

Those values could be written in the form $\{a \mid b\}$. This form does not make any quantifiable sense at the moment, because of the actual value determined by what " $a$ " and " $b$ " are.

Before explaining how to find those values, we will first consider the case in which there are no legal moves for neither players, left or right. Therefore, the player's (with no legal moves) score is left blank in the notation. If both players have no legal moves, the result is a Zero game.

\section{Definition 3.1. A Zero-game}

A Zero-game is a game that scores $\{\mid\}=0$, or in other words, the player who moves next loose, assuming all moves made are optimal.

For Hackenbush, the simplest form of a Zero game equates to an empty board at the beginning. Thus, it is obvious the first player to move has no legal move, therefore loses. In another scenario, if we were to add one blue line, left would have 0 moves after he removes the only possible line, and right would have no legal move giving left a clear 1 move advantage, written $\{0 \mid\}=1$. This trend continues in such a manner that $\{n \mid\}=n+1$, as $n$ equals the number of remaining moves left has, after making the optimal move [11].

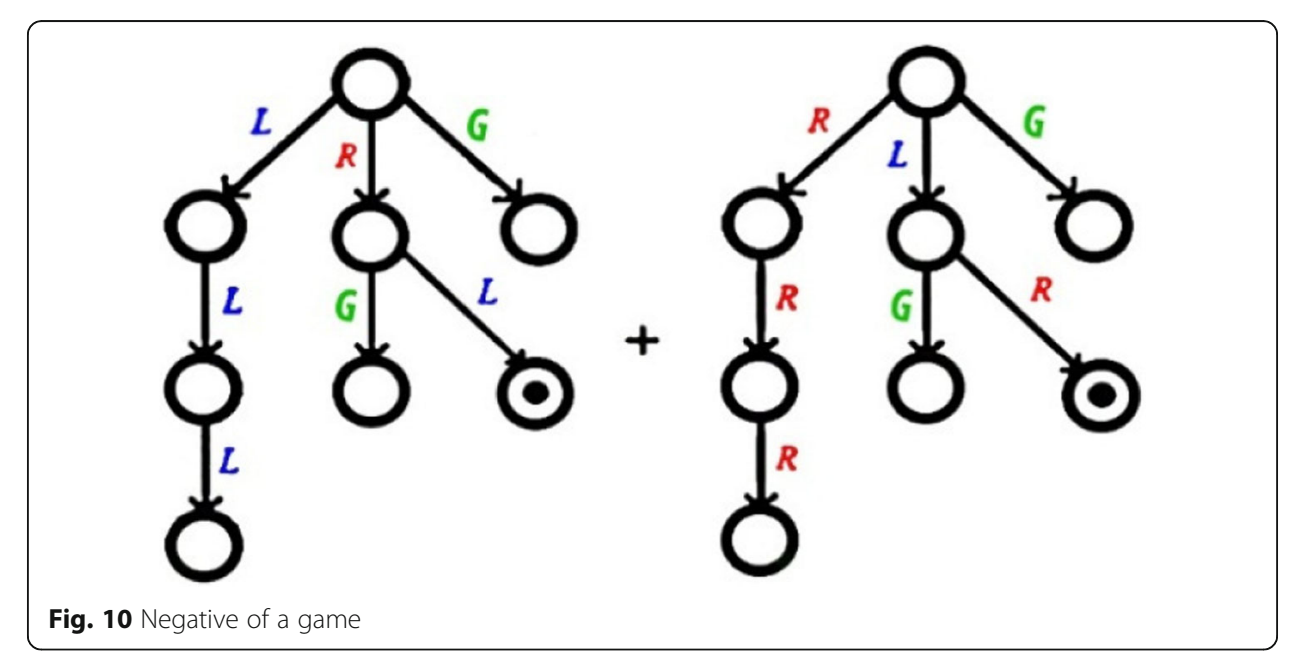


Table 1 Classification of the games by outcome

\begin{tabular}{llll}
\hline Outcome & Called & Example & Negative \\
\hline Second player wins & Zero & 0 & Zero \\
First player wins & Fuzzy & $*$ & Fuzzy \\
Left wins & Positive & 1 & Negative \\
Right wins & Negative & -1 & Positive \\
\hline
\end{tabular}

However, what will happen if we add the red line instead? As done before, these values are applied with respect to the advantage of the left player. Thus, adding one line for right results in putting left at a one move disadvantage, or a $(-1)$ advantage. So, adding one red line results in $\{\mid 0\}=-1$. Adding two red lines would then be $\{\mid-1\}=-2$. And so on to a general form of $\{\mid-n\}=-(n+1)$, as $n$ equals the number of remaining moves right has after the optimal move.

Now with the groundwork out of the way, we can start using the values of sub-games to determine the value of an overall game, for example, see Fig. 4.

After left moves, right has one legal move. In that case, we clearly have a $\{\mid 0\}=-1$ situation. Conversely, if right moves first, we have $\{0 \mid\}=1$. This results in the overall game having a value of $\{-1 \mid 1\}=0$. It makes sense because we equally added one independent move for both players to an empty board [12], which means that the advantage does not change. But what about the game in Fig. 5?

Now both players have been given one line each. But if left moves, right's only legal move is eliminated, but if right moves, left will still have one legal move. It is not so clear what the given value of this game by implementing the "scoring rules" discussed earlier. That is why we must utilize a new rule called the "simplicity rule," to assign values to the previous example [13].

Definition 3.2. $A$ *game

$\mathrm{A}$ * game (pronounced star game) is an infinitesimal game that scores.

$\{0 \mid 0\}=*$ resulting in the first player to move wins, assuming all moves made are optimal [14].

For example, Hackenbush's game has another line type that is green, which is claimable by either player. Then, we get the following game which results in a value of $\{0 \mid 0\}=*$ (see Fig. 6). Building on the previous concept, we can see results

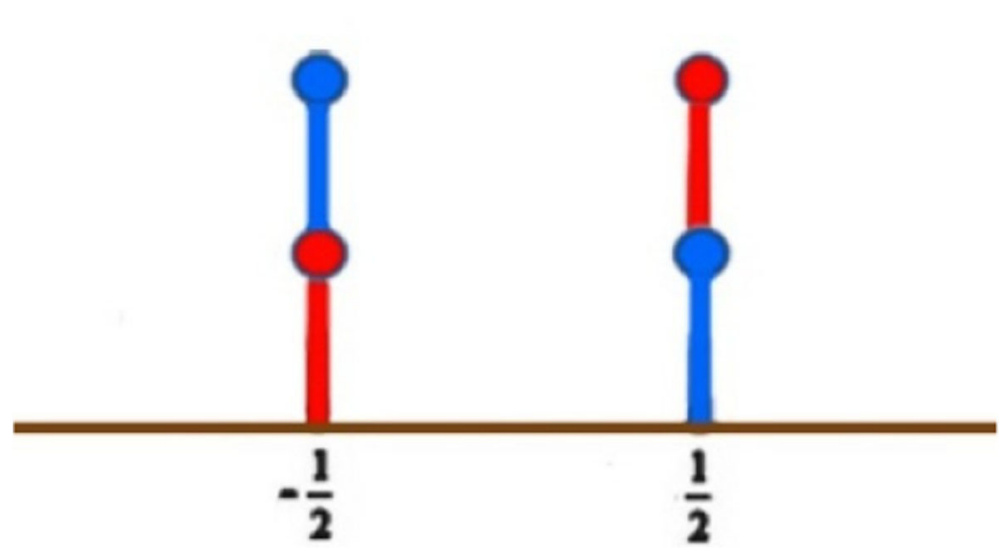

Fig. 11 Example of the inverse of $\frac{1}{2}$ 
Table 2 One to one function

\begin{tabular}{lll}
\hline Integer numbers (Z) & & Sets of classes \\
\hline & $\cdot$ & $\cdot$ \\
-2 & $\leftrightarrow$ & $\cdot$ \\
-1 & $\leftrightarrow$ & Class of -2 game \\
0 & $\leftrightarrow$ & Class of -1 game \\
1 & $\leftrightarrow$ & Class of 0 game \\
2 & $\leftrightarrow$ & Class of 1 game \\
$\cdot$ & $\cdot$ & Class of 2 game \\
. & $\cdot$ & $\cdot$ \\
\hline
\end{tabular}

like $\{n \mid-n\}=n *$ as $n *=n+*$ It is also worth noting that $*$ has the property such that $*+*=0$, see Fig. 6 .

Furthermore, there are more two infinitesimal games.

Definition 3.3. An $\uparrow$ game

An $\uparrow$-game (pronounced "up game") is a positive infinitesimal game as the score is $\{0 \mid *\}$, favoring the Left player [15], as shown in Fig. 7:

The negative version of an up game is called a down game and defined as follows.

Definition 3.4. An $\downarrow$ game

A $\downarrow$ game (pronounced "down game") is a negative infinitesimal game as the score is $\{* \mid 0\}$ which favors the right player [15].

The relation between "up" and "down" games represents the relation among the inverses of games. In every game, there is a way to reverse every move, which results in negating the value of the game originally obtained. In Hackenbush, this is obtained by replacing every red line with a blue line and vice versa. See Fig. 8 .

\section{Property 3.1.}

$$
\text { (A) }-\frac{1}{2^{\mathrm{n}}}<\downarrow<0<\uparrow<\frac{1}{2^{\mathrm{n}}}
$$

for any integer $n>0$.

(B) $\downarrow=-\uparrow$.

(C) Both $\uparrow$ and $\downarrow$ are fuzz to .

(D) $\{\uparrow \mid \downarrow\}=\{\uparrow \mid 0\}=$

$$
=\{0 \mid \downarrow\}=\{0 \mid 0\}=* \text {. }
$$

\begin{tabular}{|c|c|c|c|c|c|}
\hline+ & $\mathrm{CO} O$ & $\mathrm{CO} 1$ & $\mathrm{CO} 2$ & $\mathrm{CO} 3$ & $\mathrm{CO} 4$ \\
\hline $\mathrm{CO} O$ & CO 0 & CO 1 & $\mathrm{CO} 2$ & $\mathrm{CO} 3$ & $\mathrm{CO} 4$ \\
\hline CO 1 & CO 1 & $\mathrm{CO} 2$ & $\mathrm{CO} 3$ & $\mathrm{CO} 4$ & $\mathrm{CO} 5$ \\
\hline $\mathrm{CO} 2$ & $\mathrm{CO} 2$ & $\mathrm{CO} 3$ & $\mathrm{CO} 4$ & $\mathrm{CO} 5$ & $\mathrm{CO} O$ \\
\hline $\mathrm{CO} 3$ & $\mathrm{CO} 3$ & $\mathrm{CO} 4$ & CO 5 & $\mathrm{CO} O$ & CO 1 \\
\hline $\mathrm{CO} 4$ & $\mathrm{CO} 4$ & CO 5 & $\mathrm{CO} 0$ & CO 1 & $\mathrm{CO} 2$ \\
\hline CO 5 & CO 5 & $\mathrm{CO} O$ & CO 1 & $\mathrm{CO} 2$ & $\mathrm{CO} 3$ \\
\hline
\end{tabular}

Table 3 Addition modulo 6 in the group $H_{6}$ 
Table 4 Addition modulo 2 in the group $\left(\mathrm{H}_{2} \times \mathrm{H}_{2},+\right)$

\begin{tabular}{lllll}
\hline+ & $(0,0)$ & $(0,1)$ & $(1,0)$ & $(1,1)$ \\
\hline$(0,0)$ & $(0,0)$ & $(0,1)$ & $(1,0)$ & $(1,1)$ \\
$(0,1)$ & $(0,1)$ & $(0,0)$ & $(1,1)$ & $(1,0)$ \\
$(1,0)$ & $(1,0)$ & $(1,1)$ & $(0,0)$ & $(0,1)$ \\
$(1,1)$ & $(1,1)$ & $(1,0)$ & $(0,1)$ & $(0,0)$ \\
\hline
\end{tabular}

\section{Hackenbush group}

For every Hackenbush's game, a class can be defined. For example, the class of Zero game, see Fig. 9.

Let us define the binary addition operation to a set $(H)$ of unique classes defined above

$$
\mathrm{x}+y=\left\{x^{L}+\mathrm{y}, \mathrm{x}+y^{L} \mid x^{R}+\mathrm{y}, \mathrm{x}+y^{R}\right\}
$$

Theorem 4.1. Addition is commutative.

\section{Proof}

Base case: $G+0=0+G$ for all games $G$.

$$
\mathrm{x}+y=\left\{x^{L}+\mathrm{y}, \mathrm{x}+y^{L} \mid x^{R}+\mathrm{y}, \mathrm{x}+y^{R}\right\}=\left\{\mathrm{y}+x^{L}, y^{L}+\mathrm{x} \mid \mathrm{y}+x^{R}, y^{R}+\mathrm{x}\right\}=y+x
$$

Theorem 4.2. Addition is associative

$(\mathrm{x}+y)+z=\mathrm{x}+(y+z)$ The proof is clear.

We get negate by replacing the red $(R)$ sides with blue $(L)$ and vice versa and keep the green $(R, L)$ as it is, see Fig. 10 .

$$
-G=\left\{-G^{R} \mid-G^{L}\right\}
$$

The effect here is that all moves of left and right are switched.

$G+(-G)$ is a second player wins. For example, right first:

Right to move, so left wins.

We can classify the games by its outcomes (Table 1).

Definition 4.1. Hackenbush group

The set $H$ is defined as a group because the following conditions are met.

if $h, h_{1}, h_{2}, h_{3} \in H$

(A) Closure: if $h_{1}, h_{2}$ belong to $H, h_{1}+h_{2}$ is also in $H$.

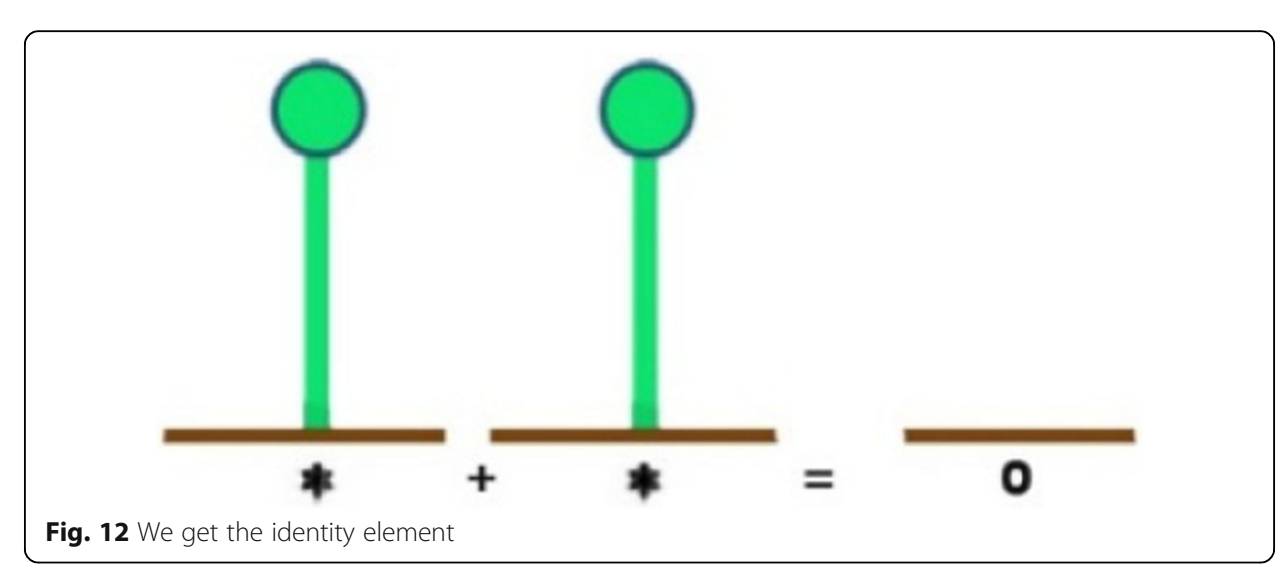


(B) Associative $h_{1}+\left(h_{2}+h_{3}\right)=\left(h_{1}+h_{2}\right)+h_{3}$ for all $h_{1}, h_{2}, h_{3}$ in $H$

(C) Identity element: there is a class 0 in $H$ such that $h+0=0+h=h$ for all $h$ in $H$

$$
\begin{aligned}
& 0+0=\{\mid\}+\{\mid\}=\{\mid\}=0 \\
& x+0=x+\{\mid\}=\left\{X_{L}+0 \mid X_{R}+0\right\}=\left\{X_{L} \mid X_{R}\right\}=x \\
& 0+y=\{\mid\}+y=\left\{0+Y_{L} \mid 0+Y_{R}\right\}=\left\{Y_{L} \mid Y_{R}\right\}=y
\end{aligned}
$$

(D) Inverse element: for each $h$ in $H$, there is an element $-h$ in $H$ such that $h+(-h)=(-h)+h=0$ see Fig. 11

(E) Commutative $h_{1}+h_{2}=h_{2}+h_{1}$ for all $h_{1}, h_{2}$ in $H$

\section{Definition 4.2. The group under addition modulo (n)}

We can create one to one function as shown in Table 2.

If we take $H_{n}=\{0,1,2, \ldots, n-1\} ; 0$ is the CO (class of) Zero games, 1 is the class of One games and so on. Let us define the operation $\oplus$ :

for every $h_{1}, h_{2} \in H_{n}$;

$$
h_{1} \oplus h_{2}=\left\{\begin{array}{cc}
h_{1}+h_{2} & h_{1}+h_{2}<n \\
h_{1}+h_{2}-n & h_{1}+h_{2} \geq n
\end{array}\right.
$$

It is clear from the definition that the operation $\oplus$ is closure, associative, commutative, and has an identity element (0). For every element $h \in H_{n}$ there is an inverse element $n-h$, or $-h=n-h$. Therefore, $H_{n}$ is a commutative group under addition modulo $n$. For example, $H_{6}=\{0,1,2,3,4,5\}$ the group under addition modulo 6 is shown in Table 3 .

The inverse of the element 1 is $-1=6-1=5$ and the inverse of 2 is $-2=6-2=4$

Example 4.1. This group $\left(2_{2} \times 2,+\right)$ consists of two tuples with addition defined element-by-element modulo 2 . An addition to the group table is shown in Table 4.

\section{What is special in Hackenbush's group?}

Hackenbush group $(H)$ is an infinite commutative group that has an element (not the identity) when added to itself, we get the identity element, see Fig. 12.

\section{Conclusion}

In this research, we have utilized combinatorial mathematical algebraic operations and concepts, through the evaluation of a deduced group from a combinatorial game called Hackenbush game to define the unique Hackenbush group.

Now, what happens if we considered the multiplication's operation on what we have analyzed above.

$$
\begin{aligned}
x y=\left\{X_{L}, X_{R}\right\}\left\{Y_{L}, Y_{R}\right\} & =\left\{X_{L} y+x Y_{L}-X_{L} Y_{L}, X_{R} y+x Y_{R}-X_{R} Y_{R} \mid X_{L} y\right. \\
& \left.+x Y_{R}-X_{L} Y_{R}, x Y_{L}+X_{R} y-X_{R} Y_{L}\right\} .
\end{aligned}
$$

Or the division's operation that is done in terms of reciprocal and multiplication 


$$
\begin{aligned}
& \frac{x}{y}=x\left(\frac{1}{y}\right) ; \frac{1}{y}= \\
& =\left\{\begin{array}{c}
0, \frac{1+\left(y^{R}-y\right)\left(\frac{1}{y}\right)^{L}}{y^{R}}, \frac{1+\left(y^{L}-y\right)\left(\frac{1}{y}\right)^{R}}{y^{L}} \mid \\
\frac{1+\left(y^{L}-y\right)\left(\frac{1}{y}\right)^{L}}{y^{L}}, \frac{1+\left(y^{R}-y\right)\left(\frac{1}{y}\right)^{R}}{y^{R}}
\end{array}\right\} .
\end{aligned}
$$

Would that lead to the creation of Rings, Fields, etc.... and would such research be applied to the world of Algebra? Only further research will tell how far Combinatorial Game Theory can go.

\section{Abbreviations}

$\uparrow$ : Up game; $\downarrow$ : Down game; CO: Class of

\section{Acknowledgements}

The authors are very thankful to the editor.

Funding

Not applicable.

\section{Availability of data and materials}

Not applicable.

\section{Authors' contributions}

All authors jointly worked on the results and they read and approved the final manuscript.

\section{Competing interests}

The authors declare that they have no competing interests.

\section{Publisher's Note}

Springer Nature remains neutral with regard to jurisdictional claims in published maps and institutional affiliations.

Received: 17 May 2018 Accepted: 15 November 2018

Published online: 12 June 2019

\section{References}

1. Kleiner, I.: The evolution of group theory: a brief survey. Math. Mag. 59, 195-215 (1986). https://doi.org/10.2307/ 2690312, ISSN 0025-570X, JSTOR 2690312

2. Smith, D.E.: History of modern mathematics, mathematical monographs no. 1 (1906)

3. Wussing, $\mathrm{H}$.: The genesis of the abstract group concept, a contribution to the history of the origin of abstract group theory. Dover Publications, ISBN 978-0-486-45868-7, New York (2007)

4. W.B.V. Kandasamy, F. Smarandache, Groups as graphs, (2009), see the link: https://arxiv.org/ftp/arxiv/papers/0906/0906.5144.pdf

5. White, A.T: Graphs of groups on surfaces: interactions and models. University of Rochester, New York (2001) Elsevier, first edition

6. Nowakowski, R.J. (ed.): Game of no chance III. Mathematical Sciences Research Institute Cambridge University Press, (2008).

7. Berlekamp, E.R., Conway, J.H., Guy, R.K.: In: Peters, A.K. (ed.) Winning ways for your mathematical plays, vol. 1, 2nd edn, Massachusetts (2001), https:/annarchive.com/files/Winning\%20Ways\%20for\%20Your\%20Mathematical\%20Plays\%20V1.pdf

8. John, S., Ryals, J.R.: Combinatorial game theory: an introduction to tree topplers, thesis under the direction of Hua Wang. Georgia Southern University, USA (2015)

9. Bouton, C.L.: Nim, a game with a complete mathematical theory. Ann. Math. 3, 35-39 (1902)

10. Conway, J.H.: Review: on numbers and games. Math. Soc. 84(6), 1328-1336 (1978)

11. McKay, N.A., Milley, R., Nowakowski, R.J.: Misère -play hackenbush sprigs. Int. J. Game Theory. 45, 731-742 (2015)

12. Siegel, A.N.: Coping with cycles. In: Games of no chance III. Cambridge University (2009), http://www.msri.org/people/ staff/levy/files/Book56/12siegel.pdf

13. Berlekamp, E.R.: The hackenbush number system for compression of numerical data. Inf. Control. 26, 134-140 (1974)

14. Fraenkel, A.: A brief biography. Electron. J. Comb, 8 (2001)

15. Albert, M., Nowakowski, R., Wolfe, D.: Lessons in play: an introduction to combinatorial game theory, CRC (2007) 